Accelerator Development Department

$\mathrm{AD} / \mathrm{RHIC}-48$ BROOKHAVEN NATIONAL LABORATORY

Associated Universities, Inc.

Upton, New York 11973

RHIC TECHNical Note No. 48

\title{
EFFECT OF THE INDUCED VOLTAGE ON THE \\ BUNCH MOTION AT THE TRANSITION ENERGY
}

S. Y. Lee and J. Wei

$3 / 20 / 89$ 


\author{
S.Y. Lee and J. Wei \\ Brookhaven National Laboratory, \\ Upton, New York 11973
}

\begin{abstract}
Analysis of the induced $160 \mathrm{MHz}$ if voltage on the bunch distribution indicates that the tolerable remnant $160 \mathrm{MHz}$ if voltage is $\mathrm{V}_{2}<25 \mathrm{KV}$ at the transition energy region. The phase space growth is found proportional to $V_{2} / V_{1}$, where $V_{1}$ is the accelerating voltage. These remnant field are not important outside the transition energy region due to synchrotron mixing. Since the bunch length in the accelerating of system is approximately $2 \pi$ radians in the high frequency if phase, the error in the high frequency if stable phase plays little role to the phase space area growth.
\end{abstract}

\section{Introduction}

For many large accelerators, there may be several if systems operating alternately. As an example, RHIC (Relativistic Heavy Ion Collider) at Brookhaven National Laboratory is proposing to use a 26.7 $\mathrm{MHz}$ rf system to accelerate the beam through the transition energy and then transfering the bunch to the $160 \mathrm{MHz}$ system for the storage collider mode in order to obtain a proper bunch length required by the experimental detector design. The $26.7 \mathrm{MHz}$ system is needed to minimize the transition energy loss due to the nonlinear if motion at the transition energy. The two rf systems are both needed in RHIC heavy ion operation.

During the acceleration stage, the $160 \mathrm{MHz}$ system may build up large induced voltage across the gap due to the beam current. The large induced field will distort the bunch structure. To minimize the effect, there are two possible solutions:

1) Operating the high frequency rf system paraphasing in pairs, so that the resulting field is $90^{\circ}$ out of phase with the accelerating if system and the magnitude of the resulting field is zero.

2) Detuning the high frequency rf cavities $\pm 2.5 f_{o}$ away from the rf resonance frequency so that the shunt impedance of the high frequency system is essentially reactive. ${ }^{2}$ Then paraphasing these rf stations in pairs can also substantially reduce the induced voltage.

Due to the accuracy of the paraphasing and the accuracy of the If phase in the high frequency if system, there is a remnant induced If voltage on the bunch motion.

Under the above operational scenario, the bunch is governed by two rf potentials, i.e. the accelerating and the induced high frequency if voltages. In either of the above scenarios, the high frequency system should track with the particle revolution frequency so that the controllable induced field can be achieved.

Normally, the high frequency if system with rf voltage less than $10 \%$ of the primary rf voltage presents little problem to the bunch motion. The bunch distribution is not much changed. The situation is different at the transition energy region due to the smallness of the synchrotron frequency. The phase space area will grow due to the action of the high frequency if system. The situation is similar to the Sorenssen effect of the space charge or inductive impedances at the transition energy region. The difference is that the space charge induced field depends on the bunch distribution, while the induced if voltage is independent of the bunch distribution in the first order.

This paper is intended to evaluate the effect of the induced voltage on the bunch distribution and evaluate the growth of the phase space area. Section 2 derives the longitudinal equation of motion for two if systems and discusses the implications. Numeri-

*Work performed under the auspices of the U.S. Department of Energy cal simulation of the bunch distribution for RHIC at the transition energy is given in section 3. The conclusion is given in section 4 .

\section{Longitudinal Equation of Motion}

Let us assume that the particle in the circular accelerators is governed by two rf systems given by

$$
V=V_{1} \sin \phi_{1}(t)+V_{2} \sin \phi_{2}(t)
$$

where $V_{1}$ and $V_{2}$ are slowly varying function of $t$ and $d \phi_{1} / d t=\omega_{1}$; $\mathrm{d} \phi_{2} / \mathrm{dt}=\omega_{2}$ are integral multiples of the particle revolution frequency $\Omega_{0}$ of the synchronous particle, i.e.

$$
\begin{aligned}
& \omega_{1}=\mathrm{h}_{1} \Omega_{0} \\
& \omega_{2}=\mathrm{h}_{2} \Omega_{0}
\end{aligned}
$$

with $\Omega_{0}=2 \pi f_{0}=\beta c / R_{0}$. Here $\beta c$ and $R_{0}$ are respectively the velocity and the average orbit radius. The energy gain per revolution for the synchronous particle is $q e\left(V_{1} \sin \phi_{1 s}+V_{2} \sin \phi_{2 s}\right)$, where qe is the charge of the particle, $\phi_{1 s}$ and $\phi_{2 s}$ are the synchronous phase of these two rf systems.

For non-synchronous particles, the deviation is defined by

$$
\Omega=\Omega_{0}+\Delta \Omega ; \phi_{1}=\phi_{1 S}+\Delta \phi_{1} ; p=p_{0}+\Delta p ; E=E_{0}+\Delta E ; \delta=\delta_{0}+\Delta \delta
$$

The phase $\phi_{2}$ of the second if system can be expressed as,

$$
\phi_{2}=\phi_{2 s}-h_{2} \Delta \theta=\phi_{2 s}+\frac{h_{2}}{h_{1}}\left(\phi_{1}-\phi_{1 s}\right)
$$

Thus the synchrotron equation of motion becomes,

$$
\frac{d \phi_{1}}{d t}=-\frac{h_{1} \eta \Omega_{0}}{P_{0} R_{0}}\left(\frac{\Delta E}{\Omega_{0}}\right)
$$

$\frac{\mathrm{d}}{\mathrm{dt}}\left(\frac{\Delta \mathrm{E}}{\Omega_{\mathrm{o}}}\right)=\frac{\mathrm{qe}}{2 \pi}\left\{\mathrm{V}_{1}\left(\sin \phi_{1}-\sin \phi_{1 s}\right)+\mathrm{V}_{2}\left(\sin \left[\phi_{2 s}+\frac{\mathrm{h}_{2}}{\mathrm{~h}_{1}}\left(\phi_{1}-\phi_{1 s}\right)\right]-\sin \phi_{2 s}\right)\right\}$

with $\eta=1 / \gamma_{\mathrm{T}}^{2}-1 / \gamma^{2}$. We have neglected the induced voltages due to the impedance of the accelerator and the space charge impedance in Eq.(5). This would simplify our interpretation of the numerical results. The effect of these impedances has been studied previously.

\section{Effect of the Induced Voltage in RHIC}

For RHIC, the proposed accelerating voltage is $V_{1}=300 \mathrm{KV}$ and $\phi_{1 \mathrm{~s}}=0.16$. The expected induced high frequency voltage is about $10 \mathrm{KV}$ at $\phi_{2 s}=0$ or $\pi$. The resulting induced if bucket is very small. The effect of the high frequency induced voltage should play 
little role in the bunch dilution due to zero acceleration in the average and the mixing of particles in the bunch due to synchrotron motion. The effect would become more important at the transition energy, where the synchrotron frequency vanishes. The bunch shape distortion here would result in the bunch area growth.

It is known that the nonlinear equation of motion is very important at the transition energy in RHIC, where Eq.(4) should be replaced by

$$
\frac{d \phi_{1}}{d t}=-h_{1} \Omega_{0}\left(\eta_{0}+\eta_{1} \delta+. .\right)
$$

with $\delta=\Delta \mathrm{p} / \mathrm{p}$ and

$$
\begin{gathered}
\eta_{0}=\frac{1}{\gamma_{T}^{2}}-\frac{1}{\gamma^{2}} \\
\eta_{1}=\frac{\eta_{0}}{\gamma_{T}^{2}}+\frac{\alpha_{1}}{2 \gamma_{T}^{2}}+\frac{3 \beta^{2}}{2 \gamma^{2}}
\end{gathered}
$$

The nonlinear term $\eta_{1} \delta$ arises from two important sources, the kinematical velocity dependence of the revolution frequency, $3 \beta^{2} / 2 \gamma^{2}$, and the property of the lattice design, ${ }^{4} \alpha_{1} / 2 \gamma^{2}$. At the transition energy region, $\eta \approx 0, \eta_{1}$ term dominates and distorts the bunch shape. The most important effect is the unstable motion, which can lead to beam loss. The effect has been shown to be detrimental for the machines at slow acceleration rates."

Besides the nonlinear effect, microwave instability due to the space charge and/or inductive impedances is noteworthy and cause phase space growth. When the nonlinear effect is combined with the microwave instability, the effect becomes even more important. Evidently, these problems can be cured by fast acceleration through the transition energy region, i.e. larger $\hat{\gamma}_{\text {or }} \hat{\gamma}_{\mathrm{T}}$ through $\gamma_{\mathrm{T}}$ jump. Once the proper $\gamma_{\mathrm{T}}$ jump is employed, the effect of the nonlinear and microwave instability can be eliminated. Presently, $\Delta \gamma_{\mathrm{T}}=0.6$ in $60 \mathrm{msec}$ is considered to be sufficient for RHIC.

To study the effect of the induced voltage, we therefore eliminate the nonlinear effect and the microwave instability by $\gamma_{T}$ jump with $\Delta \gamma_{\mathrm{T}}=0.6$ in $60 \mathrm{msec}$ by using two families of the trim quadrupoles in the arc. This justifies the neglection of the impedance terms in Eq.(5). The following parameters is used in the following calculations.

$$
\begin{array}{clr}
\mathrm{R}_{0}=610.18 \mathrm{~m} & \gamma_{\mathrm{T}}=25.4376 & \alpha_{1}=0.6 \\
\mathrm{~V}_{1}=300 \mathrm{KV} & \mathrm{V}_{2}=\text { variable } & \\
\phi_{1 \mathrm{~s}}=0.16 & \phi_{2 \mathrm{~s}}=0 \text { or } \pi \\
\mathrm{h}_{1}=342 & \mathrm{~h}_{2}=2052 &
\end{array}
$$

The phase $\phi_{2 s}$ depends on the random voltage error of the r.f. paraphasing. The magnitude $V_{2}$ in Eq.(5) depends on the accuracy of the high frequency if voltage.

For the scenario (1), operating the high frequency rf system at $300 \mathrm{KV}$ with $0.5 \%$ error in the voltage control loop in each station, we expect the total remnant voltage is $300 * 0.5 \% \sqrt{16}=6 \mathrm{KV}$.

On the other hand for the scenario (2), Each if station induces a reactive voltage of about $70 \mathrm{KV}$. for the bunch length $6 \mathrm{nsec}$. Paraphasing pairs of the if stations with frequency detuning $\pm 2.5 f_{0}$, where $f_{o}$ is the revolution frequency and assuming an accuracy of $5 \%$ variation on the reactive impedance in each cavity, we expect an induced voltage of about $14 \mathrm{KV}$.

The longitudinal equation of motion in these two scenarios is governed by Eqs.(5) and (7) with $\phi_{25}=0$ or $\pi$. Fig. 1 shows the time lapse of the bunch distribution around the transition energy region. Note here that the bunch shape distortion becomes important at the transition energy region where the synchrotron frequency becomes zero. For the phase space area of $0.3 \mathrm{eV}-\mathrm{sec} / \mathrm{amu}$, the bunch length occupies $\Delta \phi_{2} \approx 2 \pi$. Thus the bunch shape after the transition energy region would appear to be an $S$ shape or an inverted $S$ shape depending on either $\phi_{2 s}=\pi$ or 0 respectively. The phase space area would be increased by the filamentation process due to the synchrotron mixing. For $\phi_{2 s}=\pi$, which is equivalent to an inductive impedance, the bunch length is shorter at the $\gamma_{\mathrm{T}}$ region due to the combined effect of the two rf systems, the resulting phase space area growth is less than the case with $\phi_{2 s}=0$. Fig. 2 shows the tracking result of the bunch shape distortion for $\phi_{2 s}=\pi$.

Fig. 3 shows the phase space area after the $\gamma_{T}$ as a function of the strength of $V_{2}$ for $\phi_{2 s}=0$ (capacitive) and $\phi_{2 s}=\pi$ (inductive). The phase area increases linearly with $V_{2} / V_{1}$ shown on the figure. The effect is similar to that of the Sorrensen effect, where the increment of the phase area is proportional to the ratio of the space charge defocusing force and the rf focusing force. The coefficient of the proportion is slightly larger in the present case. In the Sorrensen effect, it was noted that the inductive impedance gives rise to larger phase space area growth than that of the capacitive impedance. In the case of the $\Delta$ inductive voltage with $\phi_{2 s}=0$, the growth in the phase space area is smaller.

Similar calculations can be performed to analyze the effect of the induced voltage outside the transition energy region. The synchrotron motion effectively average out the effect of the high frequency induced voltage $\left(\mathrm{V}_{2}<50 \mathrm{KV}\right)$.

\section{Conclusion}

The requirement of the two if system in RHIC, $26.7 \mathrm{MHz}$ for the particle acceleration and $160 \mathrm{MHz}$ for storage collision mode, raise the difficult problem of the induced high frequency if voltage during the bunch acceleration through the transition energy. Two possible scenarios for minimizing the damaging effect is (1) operation the $160 \mathrm{MHz}$ system paraphased in pairs so that the resulting high frequency bucket will be essential zero or (2) detuning the 160 MHz cavities $\pm 2.5 f_{0}$, where $f_{o}$ is the revolution frequency, so that the impedance of each if station is essentially reactive. Paraphasing these if stations in pairs, so that the resulting impedance of the total system is essentially zero.

However there is a small high frequency induced remnant rf voltage during the acceleration. The effect is important only at the transition energy region, where the synchrotron frequency becomes zero. The bunch will be distorted into an $\mathrm{S}$ shape distribution due to the high frequency if system. The mismatch in the bunch distribution gives rise to phase space area growth (filamentation). Our calculation shows that the tolerable high frequency of voltage is about $25 \mathrm{KV}$ for the $300 \mathrm{KV}$ accelerating of system. The expected induced of voltage is of the order of $10 \mathrm{KV}$ or smaller. Since th bunch length occupies about $2 \pi$ in the second rf phase region, the effect of the phase error (about \pm 2 degree) in the paraphasing is irrelevant.

\section{References}

\section{[1] J. Griffin, RHIC-RD-2 proposed RHIC $160 \mathrm{MHz}$ if} system.

[2] G. Cottingham, RHIC-RD-6 rf cavities and amplifiers.

[3] S.Y. Lee and J.M. Wang, IEEE-NS32, No.5, 2323,1985 . S.Y. Lee and J. Wei, to be published.

[4] E.D. Courant and H.S. Snyder, Ann. Phys. (N.Y.) 3,1,1958.

[5] S.Y. Lee and J.Wei, The nonlinear synchrotron motion in the transition region. Proc. of European Part. Acc. Conf. 1988. 


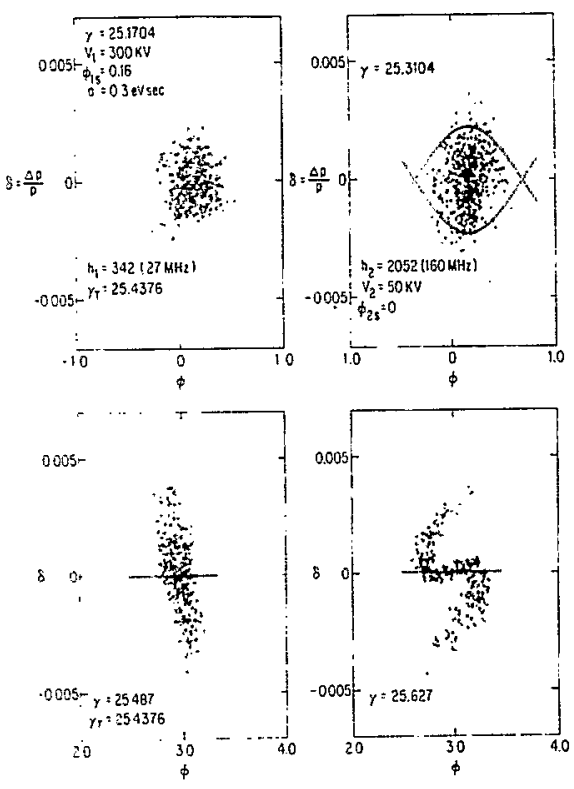

Fig. 1 The bunch distributions at $\gamma=25.1704,25.310425 .487$ and 25.627 are shown for $V_{2}=50 \mathrm{KV}$ and $\phi_{2 s}=0$ (inductive). The high frequency if bucket is shown for comparison in the second frame of the distributions. Due to the high frequency if system, the bunch distribution would becomes an $S$ shape after the transition energy crossing. Bunch dilution becomes apparent after a few synchrotron periods.
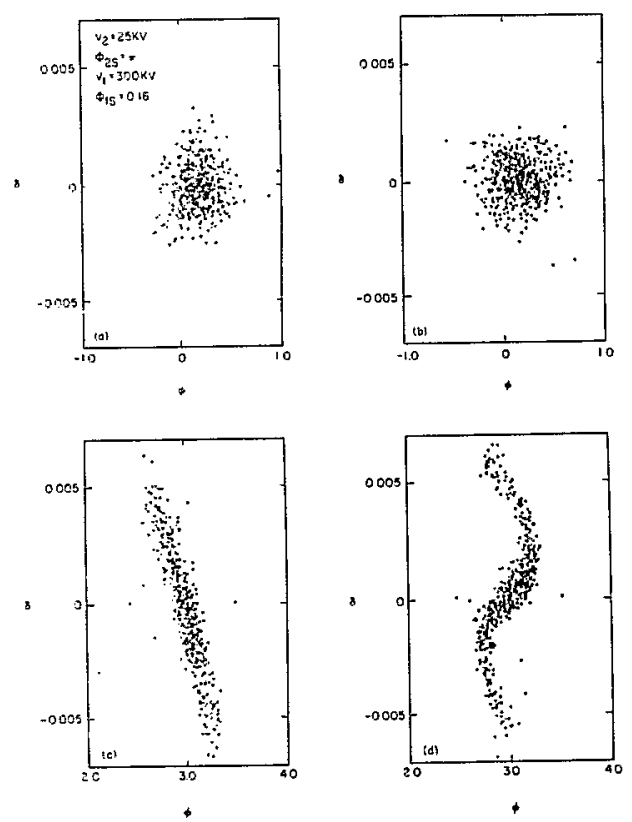

Fig. 2 Similar to that of Fig. 1 except for $\phi_{2 s}=\pi$ We observe an inverted $S$ shaped distribution after the transition energy.

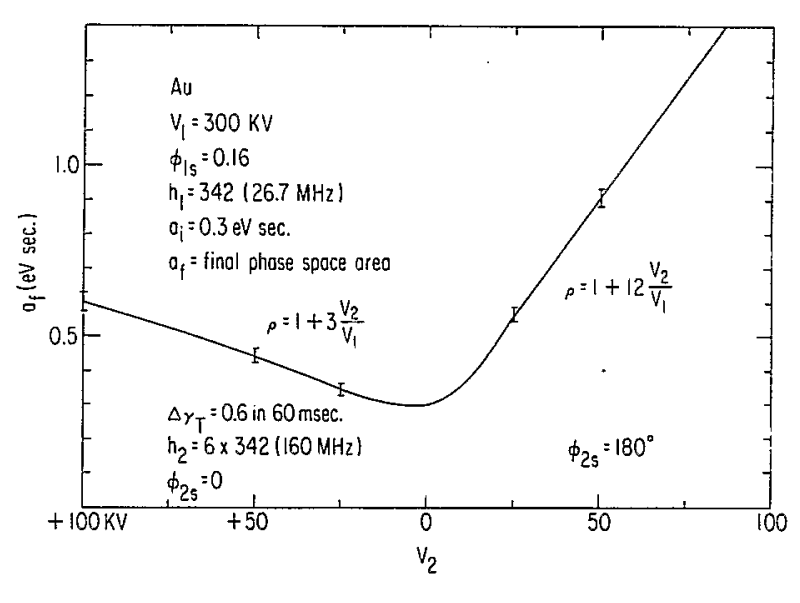

Fig. 3 Assuming an initial bunch distribution with area $a_{4}=0.3$ $\mathrm{eVsec/amu}$, the final phase space area $a_{f}$ is shown as a function of $V_{2}$ for $\phi_{2 s}=0$ (left side of $V_{2}=0$ ) and $\phi_{2 s}=\pi$ (right side of $V_{2}=0$ ). The growth factor in the phase space area $\rho=a_{p} / a_{i}$, can be fitted with a linear dependence on $V_{2} / V_{1}$ 